Ti me- of-fli ght anal yzer syst em to det ect reflected particles froma sol id surface fol l owi ng l ow energy parti cle i nj ection

\begin{tabular}{|l|l|}
\hline $\begin{array}{l}\text { jour nal or } \\
\text { publ i cat i on } \mathrm{titl} \text { e }\end{array}$ & Revi ew of Sci ent i f i c I nst r ument s \\
\hline vol une & Vol . 79 \\
\hline number & I ssue 2 \\
\hline page range & pp. 02C701- - 02C701- 4 \\
\hline year & 2008 01- 01 \\
\hline URL & ht t p: //hdl . handl e. net /10655/2199 \\
\hline
\end{tabular}




\title{
Time-of-flight analyzer system to detect reflected particles from a solid surface following low-energy particle injection ${ }^{\text {a) }}$
}

\author{
H. Yamaoka ${ }^{\text {b) }}$ \\ N. Tanaka \\ Tohoku University, Aoba, Sendai 980-8579, Japan \\ K. Tsumori and M. Nishiura \\ National Institute for Fusion Science, Toki, Gifu 509-5292, Japan \\ T. Kenmotsu \\ Kibi International University, Takahashi, Okayama 716-8505, Japan \\ T. Hirouchi, M. Kisaki, K. Shinto, ${ }^{c)}$ and M. Sasao \\ Tohoku University, Aoba, Sendai 980-8579, Japan \\ Y. Matsumoto \\ Tokushima Bunri University, Tokushima 770-8514, Japan \\ M. Wada \\ Doshisha University, Kyotanabe, Kyoto 610-0321, Japan
}

Harima Institute, RIKEN (The Institute of Physical and Chemical Research), Sayo, Hyogo 679-5148, Japan

(Received 13 August 2007; presented 27 August 2007; accepted 24 August 2007; published online 16 January 2008)

We have developed a time-of-flight analyzer to measure energy distributions of reflected particles from solid surfaces bombarded by low-energy $(1-2 \mathrm{keV})$ ions. The analyzer yields energy distributions of neutrals which can be compared with the energy distributions of charged particles measured by a magnetic deflection-type momentum analyzer. We have tested the system to measure the angular dependence of energy and intensity for neutrals reflected from a polycrystalline $\mathrm{W}$ target. The energies of the reflected neutrals are much smaller than the incident ion energies, suggesting multiple scattering in the target. No angular dependence is observed under the condition that the sum of the incident and reflected angles is constant. The intensity of the reflected neutrals takes the maximum around the mirror angle. We compare these characteristics of neutral particle reflections with those of reflected ions. (C) 2008 American Institute of Physics.

[DOI: 10.1063/1.2796173]

\section{INTRODUCTION}

Information on interactions of low-energy particles with solid surfaces is important in designing first walls and diverters for fusion reactors. In a burning plasma such as the one in ITER, the interaction between the material surface and thermalized low-energy ions in the range from a few to $10 \mathrm{keV}$ affects the overall plasma confinement and transport. The precise process of edge plasma behavior, however, has still not been fully clarified due to lack of data set for particle reflections at solid surfaces.

We have been developing an experimental system to study the fundamental processes of particle interaction with solid surfaces and used it to obtain the data set for plasmasurface interaction. ${ }^{1-5}$ With this system we have measured reflected ions for single or polycrystals ( $\mathrm{Si}, \mathrm{Mo}, \mathrm{C}, \mathrm{V}$ alloy)

\footnotetext{
${ }^{\text {a) }}$ Contributed paper, published as part of the Proceedings of the 12th International Conference on Ion Sources, Jeju, Korea, August 2007.

b) Electronic mail: yamaoka@spring8.or.jp.

${ }^{c)}$ Present address: Fusion Research and Development Directorate, Aomori Research and Development Center, Japan Atomic Energy Agency (JAEA), Rokkasho, Aomori 039-3212, Japan.
}

following the low-energy $(1-3 \mathrm{keV})$ beam injections. The angular distributions and energy spectra of positive and negative ions reflected from the materials had been measured by using a magnetic momentum analyzer. The results showed the reduction in energy of the reflected ions from the incident ion energies and a strong angular dependence of the intensity of reflected ions at a given incident angle. Similar results had been observed by other groups. ${ }^{6-9}$ We have noticed in these experiments that the reflected particles mainly consist of neutrals rather than ions and recognized the importance to measure the properties of the reflected neutral particles. Hence we employed a time-of-flight (TOF) analyzer installed complementarily to the existing magnetic momentum analyzer.

In this paper we report our system to study beam-surface interaction equipped with the TOF analyzer. As an application of the TOF analyzer, we show the results of the measurements for a polycrystalline W: angular dependences of the reflected neutral particle in their energy and intensity. We also performed the measurements of the reflected ions by the 
(a) Beam tranport system

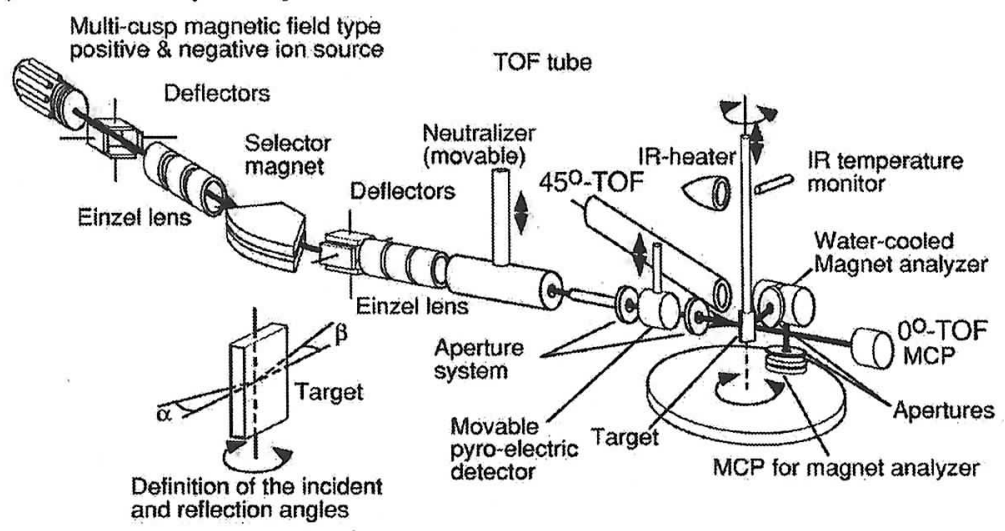

(b) Time-of-flight system

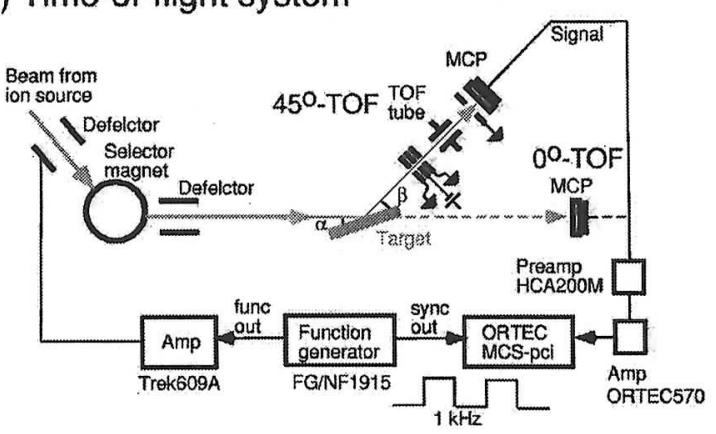

magnetic momentum analyzer. We discuss the advantage of the measurement capability for not only the reflected neutrals but also the reflected ions.

\section{BEAM TRANSPORT AND TOF MEASUREMENT SYSTEM}

Figure 1(a) shows a schematic diagram of the beam transport system. The incident angle $(\alpha)$ and reflection angle $(\beta)$ are defined in the figure. Details of the development of this system has been published elsewhere. ${ }^{3-5} \mathrm{~A}$ small multicusp magnetic-field-type ion source is used to extract positive or negative ions. Negative ions are extracted by attaching a pair of removable permanent magnets near the extraction electrodes of the ion source to realize magnetic filter geometry. We obtain $\mathrm{H}^{+}, \mathrm{H}_{2}^{+}, \mathrm{H}_{3}^{+}, \mathrm{O}^{+}, \mathrm{H}^{-}$, and $\mathrm{O}^{-}$ions. In downstream of the ion source extractor, we have the beam manipulation components; the first einzel lens, the first deflector, a bending magnet to select the ion species, a neutralizer, the second deflector, and the second einzel lens. The analyzer chamber is connected to the chamber containing ion beam production/transport system by a $50 \mathrm{~mm}$ long, $5 \mathrm{~mm}$ diameter conduit, and the two chambers are separately pumped down. The beam collimation system with apertures in front of the target defines the maximum beam size $(\phi 2 \mathrm{~mm})$.

We measure the ion beam current and the neutral beam intensity with a beam power detector located after the second einzel lens system. We can use the beam power detector with a Faraday cup mode sensing only charged particle components or a pyroelectric mode that gives total beam power
FIG. 1. (Color online) Figure 1(a) Schematic diagram of the experimental setup with the definition of incident and reflection angles. (b) Time-offlight detection. including neutrals. ${ }^{10}$ The entire reflected particle diagnostic system consists of a target, a magnet momentum analyzer with a multichannel plate (MCP) detector, two TOF tubes, and a target heating system. The angle of the target surface can be adjusted from $0^{\circ}$ to $360^{\circ}$ with respect to the incident beam, and the target can be withdrawn from the beam in the direction of the axis of target rotation. A water-cooled, $90^{\circ}$. bending magnet serving as a momentum analyzer is installed on a movable table which can rotate from $-90^{\circ}$ to $90^{\circ}$ in vacuum. The angles of the target and the magnetic momentum analyzer are controlled independently. The analyzer chamber is evacuated to better than $2 \times 10^{-6} \mathrm{~Pa}$. The incident beam intensity is measured by the beam power detector with Faraday cup mode and also monitored as the beam current onto the target. During the measurements the beam current is kept constant.

Figure 1(b) shows the TOF analyzer $\left(45^{\circ}-\mathrm{TOF}\right)$, which consists of an einzel lens system, a deflector, and a MCP with apertures. The distance from the target to the $45^{\circ}$-TOF $\mathrm{MCP}$ is about $1.6 \mathrm{~m}$. We used a fast preamplifier $(200 \mathrm{MHz}$, HCA-200M-20k-C) to form pulse signals from the MCP. The amplified MCP signal was analyzed by a multichannel scalar (ORTEC MCS-pci). The TOF system is fixed to be $45^{\circ}$ with respect to the direction of the incident beam. We measure the angular dependence of the reflected particles by changing the target angle (corresponding to change due to both the incident and reflected angles), under the condition that the sum of the incident angle $(\alpha)$ and reflection angle $(\beta)$ is constant $\left(\alpha+\beta=45^{\circ}\right)$. The low-energy ion beams of $\mathrm{H}^{+}, \mathrm{He}^{+}$, and $\mathrm{O}^{+}$ $(\leqslant 2 \mathrm{keV})$ are injected onto a $\mathrm{W}$ target. The incident beam is modulated at $1 \mathrm{kHz}$ by applying a square wave forhs 3 poten- 

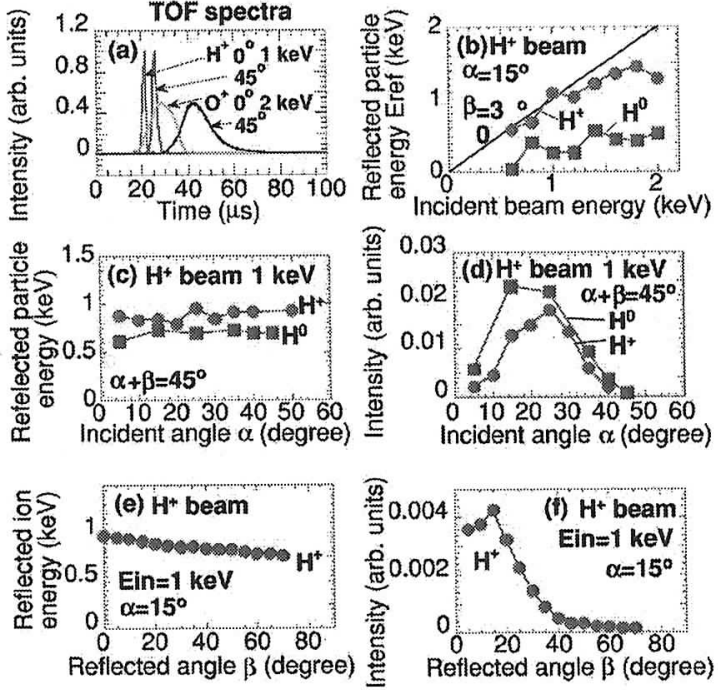

FIG. 2. (Color online) Characteristics of the reflected particles for $1 \mathrm{keV} \mathrm{H} \mathrm{H}^{+}$ beam injection on W target: (a) Examples of TOF spectra of $\mathrm{H}^{0}$ and $\mathrm{O}^{0}$ for $0^{\circ}$-TOF and $45^{\circ}$-TOF. (b) Incident beam energy dependence of the reflected particle energy at $\alpha=15^{\circ}$ and $\beta=30^{\circ}$. The solid line is proportional curve Incident angle dependences; (c) the reflected particle energies and (d) intensities under $\alpha+\beta=45^{\circ}$. Reflected angle dependences; (e) the reflected ion energy and (f) the ion intensity at $\alpha=15^{\circ}$.

tial to the deflector located at the extractor exit of the ion source. The signal for the beam modulation is also used as the trigger to a multichannel scalar. We have confirmed that the TOF signal does not depend on the modulation frequency. We have also calibrated the exact start time for the TOF analysis by a MCP locating along the primary beam axis $\left(0^{\circ}-\mathrm{TOF}\right)$. Thus we estimated the flight time from the the MCPs of $0^{\circ}$-TOF and $45^{\circ}$-TOF. We also measured the mass and incident beam energy dependence of the TOF signals. The time delay of the TOF signal is proportional to both square root of the mass and inverse square root of the beam energy, showing reliability of the system. The energy spread of the beam is measured as large as $20 \%$ for $1 \mathrm{keV}$ primary $\mathrm{O}^{+}$beam at $0^{\circ}$-TOF, while it is smaller for a lighter ion, higher energy beam. The reflected beam mainly consists of neutral particles because the intensity does not change when we apply the potential to the deflector of the $45^{\circ}$-TOF tube to remove signals due to ions. In this system we have the advantage to measure the angle and energy-resolved reflected particles of both ions and neutrals. The total reflectivity, however, cannot be directly obtained as we have not calibrated the system detection efficiencies.

\section{PARTICLE REFLECTION FROM POLYCRYSTALLINE TUNGSTEN}

Figure 2(a) shows typical TOF spectra at $0^{\circ}$-TOF and $45^{\circ}$-TOF. It should be noticed that the flight time for $45^{\circ}$ TOF is the sum of the flight time from the deflector to the target, and that from the target to the MCP. The dependence of the reflected particle energy upon the incident beam energy is shown in Fig. 2(b). The energy of reflected $\mathrm{H}^{+}$ions shows an increasing trend against the incident energy, but the energy of the reflected $\mathrm{H}^{0}$ particles saturates above the inci-
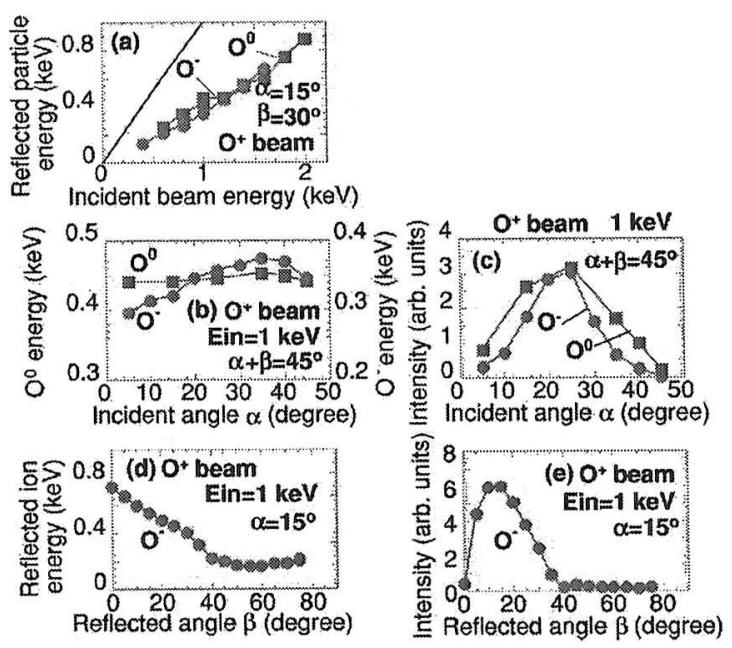

FIG. 3. (Color online) Characteristics of the reflected particles for $1 \mathrm{keV} \mathrm{O}^{+}$ beam injection on $\mathrm{W}$ target: (a) Incident beam energy dependence of the reflected particle energy at $\alpha=15^{\circ}$ and $\beta=30^{\circ}$. The solid line is proportional curve. Incident angle dependence; (b) the reflected particle energies and (c) intensities under $\alpha+\beta=45^{\circ}$. Reflected angle dependence; (d) the reflected ion energy and (e) the ion intensity at $\alpha=15^{\circ}$.

dent energy about $1.5 \mathrm{keV}$. Figures 2 (c) and 2(d) show the incident angle dependence of the reflected particle $\left(\mathrm{H}^{+}\right.$and $\mathrm{H}^{0}$ ) energy and that of the intensity under $\alpha+\beta=45^{\circ}$, respectively The reflected particle energies do not depend on the incident angle $\alpha$ under $\alpha+\beta=45^{\circ}$. The mean energy of the reflected ions is larger than that of the neutrals. We have observed that the reflected neutral particle energy has shown a time dependence during $\mathrm{H}^{+}$injection. This may be attributable to the retention of hydrogen in W. Further study is required for this problem. The intensities take maxima around the mirror angle. Figures 2(e) and 2(f) show the dependence on the reflection angle of the reflected $\mathrm{H}^{+}$ion energy and that of intensity at $\alpha=15^{\circ}$. The reflected ions have strong dependence on the reflection angle $\beta$, and the intensity shows the maximum around the angle of mirror reflection.

A similar measurement has been performed for $\mathrm{O}^{+}$ion injection, as shown in Fig. 3. The reflected ions mainly consist of negative $\mathrm{O}^{-}$ions. The energies of the reflected $\mathrm{O}^{-}$ions and neutral $\mathrm{O}^{0}$ particles are nearly the same with linear dependences against the incident beam energy, as shown in Fig. 3(a). The reflected energies of $\mathrm{O}^{-}$and $\mathrm{O}^{0}$ exhibit weak angular dependences showing maxima around the same angle of $25^{\circ}$ under $\alpha+\beta=45^{\circ}$, corresponding to the mirror reflection condition. The mean reflected ion energy showed a decreasing characteristic against the reflection angle $\beta$, as shown in Fig. 3(d). Figure 3(e) shows that the energy of the reflected $\mathrm{O}^{+}$ions takes the maximum around the mirror reflection angle. Compared to the cases of $\mathrm{H}^{+}$injection, the ratio of the reflected particle energy to the incident ion energy is smaller, and the dependence on the reflected angle for the reflected ion intensity is stronger. This may be due to the increase of the elastic energy loss with the larger mass.

We have calculated these processes by using the Monte Carlo simulation atomic collision in amorphous target code for neutral particle reflection. ${ }^{11}$ The results show th132 2 or an 
example, the energy of the reflected $\mathrm{H}^{0}$ particles reduced from $90 \%$ at $\alpha=5^{\circ}$ to $50 \%$ at $\alpha=40^{\circ}$ for $1 \mathrm{keV} \mathrm{H} \mathrm{H}^{+}$beam injection on the $\mathrm{W}$ target. The reflected particle energy is almost constant for the change in the incident angle $\alpha$ under $\alpha+\beta=45^{\circ}$. Similar results are obtained for $\mathrm{He}^{+}$and $\mathrm{O}^{+}$injections. The results of these calculations support our experimental results. The measured energies of the reflected neutral particles for $\mathrm{O}^{+}$injection, however, are less than the calculated values. Comparison of our experimental results with the simulation suggests the presence of multiple scattering in the surface collision process. The simulation results show several tens of collision events in the material. For light atom injection such as $\mathrm{H}^{+}$, the inelastic energy loss is dominant, but for $\mathrm{He}^{+}$and $\mathrm{O}^{+}$, it is comparable to the elastic energy loss. The actual surface is not perfectly flat but has a finite surface roughness. A fractal dimension between two and three should be taken into account to explain the effect of surface roughness to compare the result with the experimental ones qualitatively. ${ }^{12}$ This work is now under progress.

In this paper we describe the TOF system for measurement of reflected particles from the surface. The system is developed complementarily for the magnetic momentum analyzer which can measure only charged components in reflected particles. As the demonstration of the system capability, the results of the energy and angular dependences of reflected particles are shown for polycrystalline $\mathrm{W}$ target. We have compared the energy distributions of reflected neutrals measured by TOF analyzer with those of ions measured by magnetic momentum analyzer. Reflected neutrals seem to possess smaller energies than ions, indicating the effect of multiple scattering in the target. We continue both experimental and simulation studies to further deepen our understanding on the final charge state of the reflected particle.

\section{ACKNOWLEDGMENTS}

This work was performed under the collaboration program of NIFS [No. 143 (2006) and No. 142 (2007)].

${ }^{1}$ M. Wada, M. Sasao, M. Nishiura, H. Yamaoka, and Y. Matsumoto, Rev. Sci. Instrum. 73, 955 (2002).

${ }^{2}$ M. Sasao, Y. Matsumoto, A. Mendenilla, M. Nishiura, K. Shinto, M. Wada, and H. Yamaoka, Proceedings of the 70th European Physical Society Conference on Controlled Fusion and Plasma Physics, St. Petersburg, July 2003 (unpublished), Vol. 27A, paper No. 2.161.

${ }^{3}$ H. Yamaoka, Y. Matsumoto, M. Nishiura, M. Sasao, and M. Wada, Rev. Sci. Instrum. 337-339, 942 (2005)

${ }^{4}$ H. Yamaoka, Y. Matsumoto, M. Nishiura, K. Tsumori, H. Sugawara, S. Takeuchi, K. Shinto, M. Sasao, and M. Wada, Rev. Sci. Instrum. 77, 03C301 (2006).

${ }_{5}^{5}$ H. Yamaoka, N. Tanaka, Y. Matsumoto, M. Nishiura, K. Tsumori, H Sugawara, S. Takeuchi, K. Shinto, A. Okamoto, M. Sasao, and M. Wada, J. Nucl. Mater. 363-365, 1304 (2007).

${ }^{6}$ H. Verbeek, W. Eckstein, and S. Datz, J. Appl. Phys. 47, 1785 (1976).

${ }^{7}$ H. Verbeek, W. Eckstein and R. S. Bhattacharya, Surf. Sci. 95, 380 (1980)

${ }^{8}$ R. Aratari and W. Eckstein, Nucl. Instrum. Methods Phys. Res. B 42, 11 (1989).

${ }^{9}$ H. Niehus, W. Heiland, and E. Taglauer, Surf. Sci. Rep. 17, 213 (1993).

${ }^{10}$ A. Viehl, M. Kanyo, A. van der Hart, and J. Schelten, Rev. Sci. Instrum. 64, 732 (1993)

${ }^{11}$ Y. Yamamura, Nucl. Instrum. Methods Phys. Res. B 51, 407 (1990).

${ }^{12}$ T. Kenmotsu, Y. Yamamura, T. Muramoto, and N. Hirotani, Nucl. Instrum. Methods Phys. Res. B 228, 369 (2005) 